\title{
Mitochondrial transcription factor A contributes to cisplatin resistance in patients with estrogen receptor-positive breast cancer
}

\author{
WEI GAO, MEI-HONG WU, NING WANG, MING-ZHENG YING, \\ YING-YI ZHANG, JING HUA, LIU CHUAN and YA-JIE WANG \\ Department of Oncology, Changhai Hospital, Second Military Medical University, Shanghai 200433, P.R. China
}

Received September 13, 2015; Accepted September 19, 2016

DOI: $10.3892 / \mathrm{mmr} .2016 .5881$

\begin{abstract}
Previous studies have reported that triple-negative breast cancer is more sensitive to cytotoxic treatment, compared with estrogen receptor (ER)-positive cancer. However, the underlying molecular mechanisms remain to be fully elucidated. In the present study, we employed reverse transcription-quantitative polymerase chain reaction, western blot and in vivo assays to investigate the underlying mechanisms. The sensitivities of cells to cisplatin were examined in ER-positive and ER-negative breast cancer cells, and it was found that the ER-negative cells were more sensitive to cisplatin, compared with the ER-positive cells. In addition, it was found that mitochondrial transcription factor A (TFAM), which functions in mitochondrial DNA replication and repair, was expressed at a high level in ER-positive cell lines and patient tissues, compared with ER-negative cell lines and tissues. It was also found that the sensitivity to cisplatin was decreased when TFAM was knocked down in the breast cancer cells, and these effects were reversed when TFAM was reintroduced to the cells. Similar results were observed in xenograft tumors. The results of the present study provided evidence that resistance to cisplatin chemotherapy in ER-positive breast cancer may be through TFAM and indicated that TFAM may be a target for chemoresistance in patients with breast cancer. These findings offer potential guidance for chemotherapy in patients with breast cancer.
\end{abstract}

\section{Introduction}

Breast cancer is the most common type of cancer diagnosed among women and, in America, one in eight women develop

Correspondence to: Professor Ya-Jie Wang, Department of Oncology, Changhai Hospital, Second Military Medical University, 168 Changhai Road, Shanghai 200433, P.R. China

E-mail: yajiewang_chh@163.com

Key words: breast cancer, estrogen receptor, mitochondrial transcription factor A, cisplatin resistance breast cancer in their lifetime, with breast cancer accounting for almost one in eight cases of cancer and being the second leading cause of cancer-associated mortality among women (1). Although there have been advances in breast cancer diagnosis and treatment, the molecular mechanisms underlying breast cancer remain to be fully elucidated, particularly regarding chemotherapy resistance (2).

It is known that cisplatin is one of the most potent antitumor agents, exhibiting clinical activity against a wide variety of solid types of tumor, including breast cancer (3). Cisplatin can interact with nuclear DNA and mitochondrial DNA (mtDNA) to form DNA adducts, primarily intrastrand crosslink adducts, which activate several signal transduction pathways, including those involving ATR, p53, p73 and MAPK, which culminate in the activation of apoptosis (4-7). However, clinical studies have shown that patients with estrogen receptor (ER)-positive cancer are more likely to develop cisplatin resistance $(8,9)$. These findings suggest that estrogen may protect breast cancer cell from cisplatin insult through activating ER.

Previous studies have shown that estrogen can bind to $\mathrm{ER} \alpha$ and $\beta$ to stimulate the transcription of nuclear respiratory factor 1 (NRF1), and the NRF1 transcription factor can regulate the transcription of mitochondrial transcription factor $\mathrm{A}$ (TFAM) $(10,11)$. TFAM is essential in mtDNA replication and repair $(12,13)$. It is known that cisplatin can destroy mtDNA to trigger cell death signaling (14). Therefore, the present study hypothesized that estrogen may activate ER and then elevate the expression of TFAM to promote cell survival from cisplatin treatment.

\section{Materials and methods}

Patients and ethics statement. A total of 16 fresh ER-positive breast cancer tissues and 16 triple-negative breast cancer tissues were collected from patients with pathologically and clinically confirmed breast cancer. The tissues were collected from patients with pathologically and clinically confirmed breast cancer. The age of these women patients ranged from 27 to 55 years old, and were collected from the November 2012 to December 2014 , and the sizes ranged from $0.2 \times 0.7 \mathrm{~cm}$ to $2.4 \times 2.6 \mathrm{~cm}$. All human tumor tissues were obtained with 
written informed consent from the patients. The Institutional Review Board of Cangzhou Central Hospital (Cangzhou, China) approved the use of the tumor samples in the present study.

Cell culture. MCF-7, T47D, MDA-MB-231 and MDA-MB-453 cell lines were purchased from America Type Culture Collection (ATCC; Manassas, VA, USA). All cells were maintained under standard culture conditions of $37^{\circ} \mathrm{C}$ and $5 \%$ $\mathrm{CO}_{2}$ in Dulbecco's modified Eagle's medium (Gibco; Thermo Fisher Scientific, Inc.), as recommended by ATCC.

RNA isolation and reverse transcription-quantitative polymerase chain reaction $(R T-q P C R)$ analysis. Total RNA was purified from homogenized breast cancer tissues or cells using TRIzol reagent (Invitrogen; Thermo Fisher Scientific, Inc., waltham, MA, USA) following the manufacturer's protocol. The RNA ( $2 \mu \mathrm{g})$ was reverse transcribed using SuperScript Reverse Transcriptase III (Invitrogen; Thermo Fisher Scientific, Inc.). qPCR analysis was performed using SYBR green Supermix (ABI; Thermo Fisher Scientific, Inc.) in an ABI 7900 PCR system (ABI; Thermo Fisher Scientific, Inc.). All the procedures were performed according to the manufacturer's recommended protocol. Reactions were incubated at $95^{\circ} \mathrm{C}$ for $30 \mathrm{sec}$, followed by 40 cycles at $95^{\circ} \mathrm{C}$ for $5 \mathrm{sec}$ and $60^{\circ} \mathrm{C}$ for $31 \mathrm{sec}$, and finally $95^{\circ} \mathrm{C}$ for $15 \mathrm{sec}, 60^{\circ} \mathrm{C}$ for $1 \mathrm{~min}$ and $95^{\circ} \mathrm{C}$ for $15 \mathrm{sec}$. The relative expression of NRF1 and TFAM was calculated using the $2^{-\Delta \Delta \mathrm{Cq}}$ method (15) with GAPDH RNA as the reference gene. The housekeeping gene, GAPDH, was used as an internal standard. The primers using in the present study are listed in Table I.

Western blot analysis. The cells were lysed in WB/IP lysis buffer (cat. no. P0013; Beyotime Institute of Biotechnology, Haimen, China) and nuclear proteins were extracted using lysis buffer (cat. no. P0028; Beyotime Institute of Biotechnology). All procedures were performed following the manufacturer's protocol. Protein concentrations were determined by bicinchoninic acid assay. Subsequently, the cell lysates were boiled in 5X SDS-PAGE loading buffer for $10 \mathrm{~min}, 30-50 \mu \mathrm{g}$ samples were resolved by $8 \%$ SDS-PAGE and then transferred onto nitrocellulose membranes. The membranes were incubated with the following antibodies at $4^{\circ} \mathrm{C}$ overnight $(1: 1,000$ dilution): NRF1 (cat. no. 12482-1-AP; ProteinTech Group, Inc.; Chicago, IL, USA), TFAM (cat. no. 19998-1-AP; ProteinTech Group, Inc.), ER $\alpha$ (cat. no. 21244-1-AP; ProteinTech Group, Inc.) and GAPDH (cat. no. 2118; CST Biological Reagents Company Limited, Shanghai, China). Subsequently, the membranes were incubated with anti-rabbit (cat. no. 5127) and anti-mouse (cat. no. 58802) secondary antibodies (1:2,000 dilution; Cell Signaling Technology, Inc., Danvers, MA, USA). The bound antibodies were visualized with an ECL kit (Thermo Fisher Scientific, Inc.).

Construction of stable cell lines. To generate stable TFAM-silenced cell lines, vectors containing short hairpin (sh)RNAs were purchased from Sigma-Aldrich; Merck Millipore (Darmstadt, Germany). 293T cells (ATCC) were transfected with these vectors using Lipofectamine 2000 (Invitrogen; Thermo Fisher Scientific, Inc.) with all procedures
Table I. Sequence of primers and shRNAs.

\begin{tabular}{ll}
\hline Primer/shRNA & \multicolumn{1}{c}{ Sequence (5'-3') } \\
\hline NRF1 & F: GGTCGCAGTCTCCACGG \\
& R: ATGTTCGGTTTGGGTCACTC \\
GAPDH & F: AGCCTCAAGATCATCAGCAATGCC \\
& R: TGTGGTCATGAGTCCTTCCACGAT \\
TFAM & F: TCCTCTCCAAAATGCCAGAG \\
& R: TCCAGTTTCCTTTACAGTCTTCAG \\
Sh-\#1 & CCGGCGTGAGTATATTGATCCAGAAC \\
& TCGAGTTCTGGATCAATATACTCACG \\
& TTTTTG \\
CCG-\#2 & CGAGAAAATCGATCTTACCTTCGATTTCT \\
& TTTTTG
\end{tabular}

shRNA, short hairpin RNA; NRF1, nuclear respiratory factor 1 TFAM, mitochondrial transcription factor A; F, forward; R, reverse.

performed according to the manufacturer's protocols. The supernatant media containing the virus was collected by centrifugation $\left(1.2 \times 10^{5} \mathrm{x} g\right.$ for $\left.10 \mathrm{~min}\right)$ to remove cellular debris. The viruses were used to infect the indicated cells, and the transfected cells were then selected by exposure to $2 \mu \mathrm{g} / \mathrm{ml}$ puromycin for 2 weeks. The alterations of TFAM in these cells were confirmed using PCR and western blot analysis prior to further analysis. The sequences of the shRNAs used are listed in Table I. As Sh-\# 2 showed a lower relative efficiency, Sh-\#1 was selected to perform the subsequent experiments.

Overexpression of TFAM. The constructed stable cell lines were transfected with pCDNA3.1 (Thermo Fisher Scientific, Inc.), which contained the open reading frame of TFAM, using Lipofectamine 2000 (Invitrogen; Thermo Fisher Scientific, Inc.) to re-introduce TFAM to these cell lines. All procedures were performed according to the manufacturer's protocol.

Cell Counting Kit-8 (CCK-8) cell viability assays and caspase 3/7 apoptosis assays. The cells were seeded into a 96-well plate at a density of $3 \times 10^{3}$ cells per well with $100 \mu \mathrm{l}$ culture medium and MCF-7 cells were seeded in 6 -well plates at a density of 300,00 cells/well, and then cells were placed in phenol-red media supplemented with $5 \%$ dextran-charcoal-stripped fetal bovine serum (Gibco; Thermo Fisher Scientific, Inc.), MEM nonessential amino acids (Gibco; Thermo Fisher Scientific, Inc.), gentamicin (MCE China, Shanghai, China) and $6 \mathrm{ng} / \mathrm{ml}$ bovine insulin (YuanYe Biotechnology Co., Ltd., Shanghai, China) for $48 \mathrm{~h}$ at $37^{\circ} \mathrm{C}$. The cells were then treated with estradiol ( $0.1 \mathrm{nM}$; Selleck Chemicals, Houston, TX, USA) or estradiol and fulvestrant (6 $\mu \mathrm{g} / \mathrm{ml}$; Selleck Chemicals) for $24 \mathrm{~h}$ at $37^{\circ} \mathrm{C}$, following which and the medium was replaced and the cells were treated with the indicated concentrations $(0,2,5,10,15$ and $20 \mu \mathrm{M}$ ) of cisplatin (Sigma-Alrich; Merck Millipore) for $14 \mathrm{~h}$ at $37^{\circ} \mathrm{C}$. The cell viabilities were then quantified by the addition $10 \mu \mathrm{l}$ of CCK-8 (Dojindo Molecular Technologies, Inc., Kumamoto, Japan). Following incubation for $1.5 \mathrm{~h}$ at $37^{\circ} \mathrm{C}$, 
A
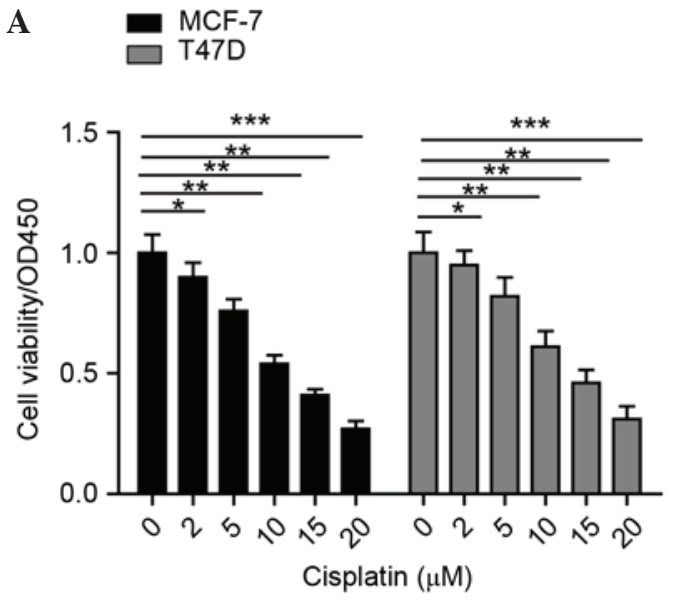

C

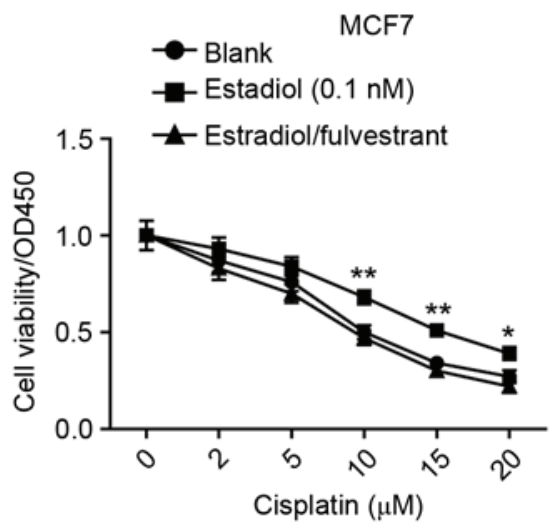

B
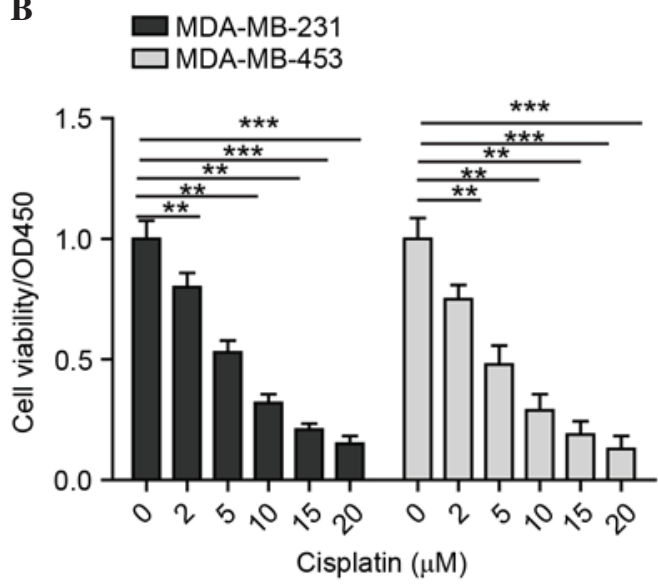

D

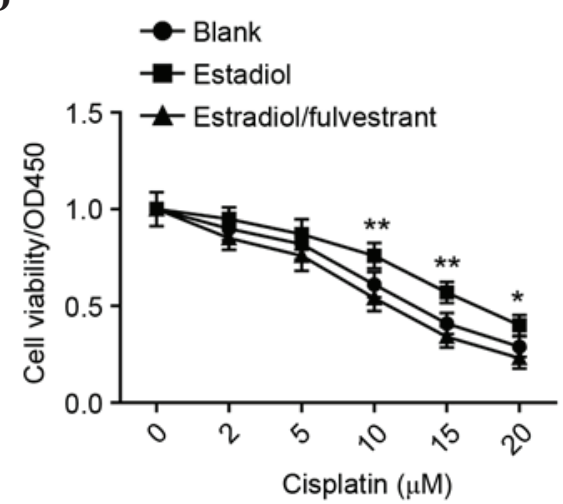

Figure 1. ER-positive breast cancer cell lines are more sensitive to cisplatin. (A) ER-positive MCF-7 and T47D breast cancer cell lines, and (B) ER-negative MDA-MB-231 and MDA-MB-453 breast cancer cell lines were treated with the indicated concentrations of cisplatin for $24 \mathrm{~h}$. (C) MCF-7 cells and (D) T47D cells were pre-incubated with estradiol or estradiol and fulvestrant for $24 \mathrm{~h}$, and then treated with the indicated concentrations of cisplatin for another $24 \mathrm{~h}$. ${ }^{*} \mathrm{P}<0.05,{ }^{* *} \mathrm{P}<0.01$ and ${ }^{* * *} \mathrm{P}<0.001$. All experiments were performed in triplicate. ER, estrogen receptor; OD, optical density.

the plates were monitored using a Power Wave XS microplate reader (BioTek Instruments, Inc., Winooski, VT, USA) at an absorbance $450 \mathrm{~nm}$. Cell apoptosis assays were performed using a Caspase3/7 Glo kit (Promega Corporation, Madison, WI, USA) and all procedures were performed according to the manufacturer's protocols.

Tumorigenesis in vivo. All mice $(\mathrm{n}=48)$ were purchased from Shanghai Laboratory Animal Center (Shanghai, China). All the mice were 8 week-old females and were housed at $25^{\circ} \mathrm{C}$ with access to sterile water and food. A total of $1.0 \times 10^{6}$ of the stably transfected MCF-7 cells (Sh-\#1 or negative control shRNA) were implanted subcutaneously into the right flank of BALB/c (nu/nu) mice. At the same time, estradiol $(0.06 \mathrm{mg})$ or estradiol and fulvestrant $(0.06 \mathrm{mg})$ were injected every 2 days for 2 months (eight in each treatment group). After the 2 months, cisplatin was injected at a dose of $10 \mathrm{mg} / \mathrm{kg}$ body weight every 2 days for 2 months. After 8 weeks, the mice were sacrificed by cervical dislocation for the analysis of tumor burden. All procedures were performed in accordance with The Animal Care and Use Committee of the Second Military Medical University (Shanghai, China).

Statistical analysis. Data are expressed as the mean \pm standard deviation. Analysis was performed using SPPS software (version 19; IBM SPSS, Armonk, NY, USA) Two tail student's $t$-test was used for comparisons between control and test groups. $\mathrm{P}<0.05$ was considered to indicate a statistically significant difference.

\section{Results}

ER-positive breast cancer cells are more sensitive to cisplatin. Clinical studies have shown that patients with triple-negative breast cancer are more sensitive to cisplatin chemotherapy, compared with patients with non-triple-negative breast cancer, particularly those with ER-positive cancer (16). This suggests that ER may be essential in cisplatin chemoresistance, and the present study was performed to investigate the underlying mechanism. In the present study, ER-positive MCF-7 and T47D breast cancer cell lines, and ER-negative MDA-MB-231 and MDA-MB-453 breast cancer cell lines (17-20) were selected to detect their sensitivies to cisplatin. All cell lines were treated with the indicated concentrations of cisplatin for $24 \mathrm{~h}$, and their viabilities were examined using CCK-8 assays. As shown in Fig. 1A and B, the results confirmed the clinical observation; ER-negative cells were more sensitive to cisplatin treatment. These findings suggested that ER has an aggressive role in the chemoresistance of cells to cisplatin. Subsequently, MCF-7 and MDA-MB-231 cell lines were selected to examine the reactions when the cells were treated with estradiol or with estradiol and fulvestrant, an ER 
A
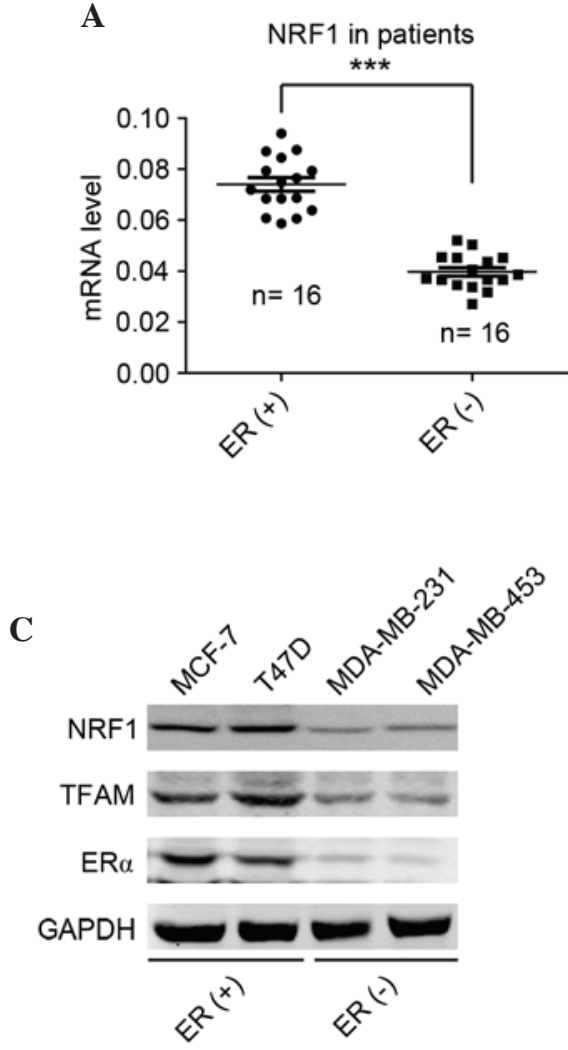

B
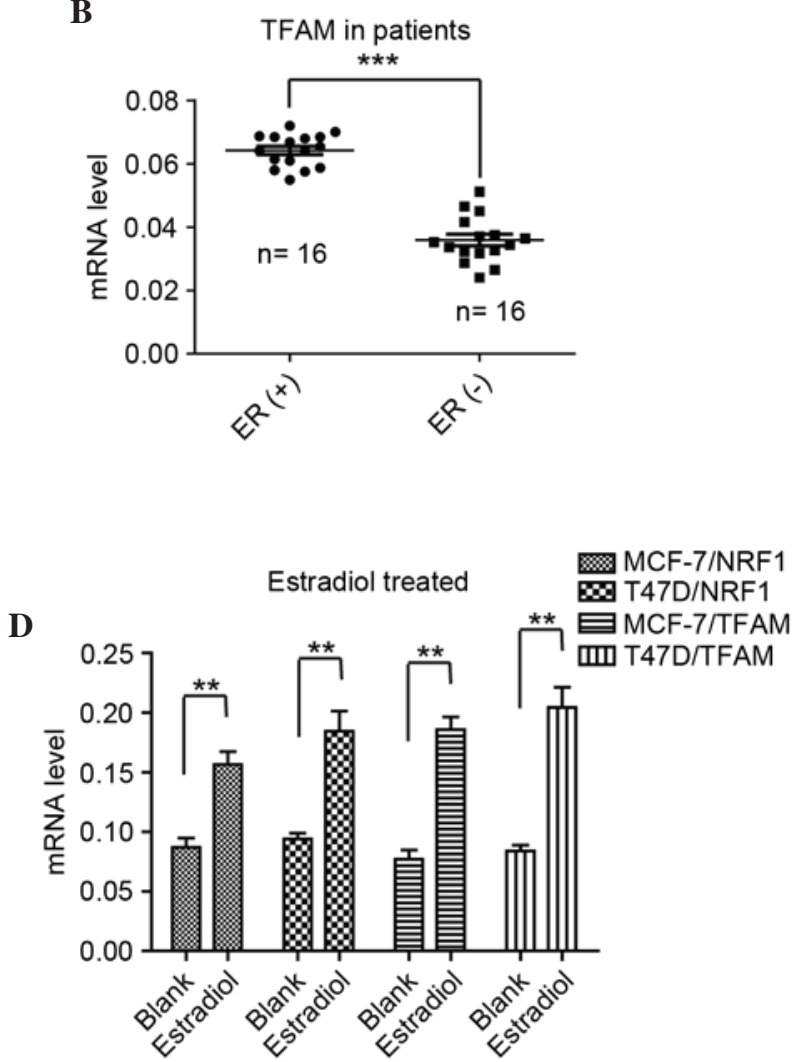

Figure 2. Levels of TFAM are positively correlated with NRF1 and ER. The mRNA levels of (A) NRF1 and (B) TFAM were examined in 16 ER-positive and 16 ER-negative fresh tissues from patients. (C) Protein levels of NRF1, TFAM and ER $\alpha$ were examined in four breast cancer cell lines. (D) mRNA levels of NRF1 and TFAM in MCF-7 and MDA-MB-231 cells following treatment with estradiol for 24 h. ${ }^{* *} \mathrm{P}<0.01$ and ${ }^{* * * *} \mathrm{P}<0.001$. ER, estrogen receptor; ER (+), ER-positive cell; ER (-), ER-negative cell; NRF1, nuclear respiratory factor 1; TFAM, mitochondrial transcription factor A.

inhibitor. As shown in Fig. 1C and D, it was found that, when treated with estradiol, the MCF-7 and T47D cells exhibited a higher tolerance to the different concentrations of cisplatin, and this increased tolerance was reversed when the cells were incubated with estradiol and fulvestrant. Taken together, these results confirmed the oncogenic role of ER in cisplatin resistance in breast cancer.

Levels of TFAM are positively correlated with NRF1 and $E R$. The mechanism underlying the effect of ER in cisplatin resistance in breast cancer remains to be fully elucidated. A previous report provided evidence to suggest that NRF1 may be essential during the chemoresistance process in breast cancer (21). In the present study, the mRNA level of NRF1 was detected in 18 ER-positive patient tumor tissues and 18 ER-negative tissues. As shown in Fig. 2A, the expression levels of NRF1 were significantly higher in the ER-positive tissues, compared with the ER-negative tissues. The expression pattern of NRF1 in the breast cancer tissues prompted investigation to further elucidate the role of NRF1 in cisplatin resistance in breast cancer. NRF1 functions as a transcription factor and previous reports on the functions of NRF1 have revealed that TFAM, which encodes a key mitochondrial transcription factor, and is essential in mtDNA replication and repair, is usually transactivated by NRF1 (11). As is already known, cisplatin functions to destroy the double strand of DNA and mtDNA is usually damaged by cisplatin (22). The present study hypothesized that a high expression level of
TFAM may protect ER-positive breast cancer cells from the insult of cisplatin (4). The present study detected the mRNA levels of TFAM in the same two groups of breast cancer tissues, and found that the levels of TFAM in the ER-positive tissues were significantly higher, compared with those in the ER-negative tissues (Fig. 2B). Furthermore, it was found that the expression of TFAM was positively correlated with the level of NRF1 in the ER-positive breast cancer tissues (Fig. 2B). To confirm the results from the breast cancer tissues, the present study examined the protein levels of NRF1, TFAM and ER $\alpha$ in the ER-positive and ER-negative cell lines. As shown in Fig. 2C, it was found that the protein levels of NRF1 and TFAM were positively correlated with the expression of ER $\alpha$, and that NRF1 and TFAM were expressed at high levels in the MCF-7 and T47D cells, and relatively lower in the MDA-MB-231 and MDA-MB-453 cells. To further validate the associations among ER, NRF and TFAM, the ER-positive MCF-7 and T47D cells were selected to examine the alterations when treated with estradiol. As shown in Fig. 2D, the levels of NRF1 and TFAM mRNA were significantly elevated when the cells were treated with estradiol. These findings indicated that estradiol activated ER and then transactivated the transcription of NRF1, followed by the elevated expression of TFAM to promote ER-positive breast cancer cell survival from cisplatin therapy.

TFAM knockdown elevates sensitivity to cisplatin in ER-positive breast cancer cells. The present study further investigated whether TFAM regulates the sensitivity of 
A

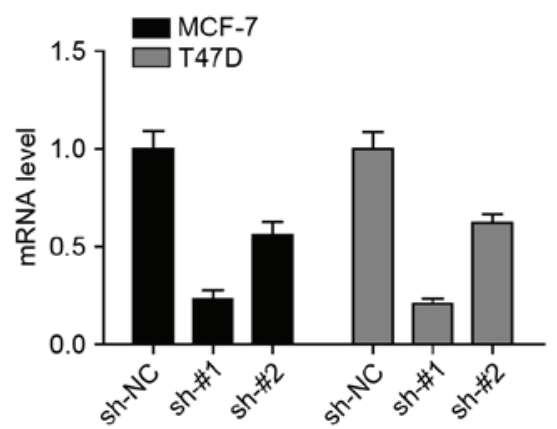

C

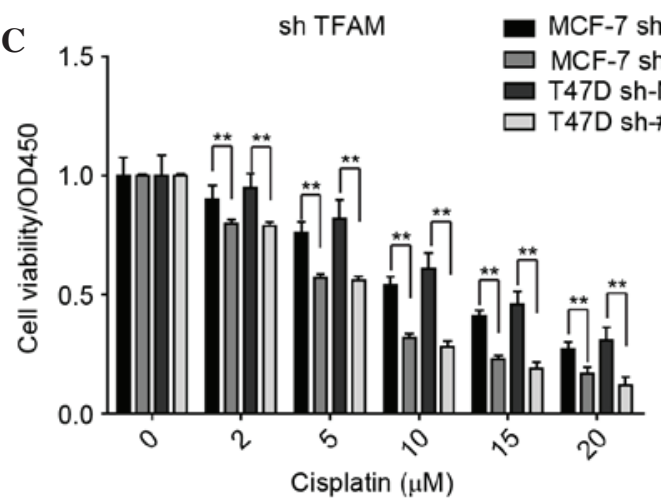

$\mathbf{E}$

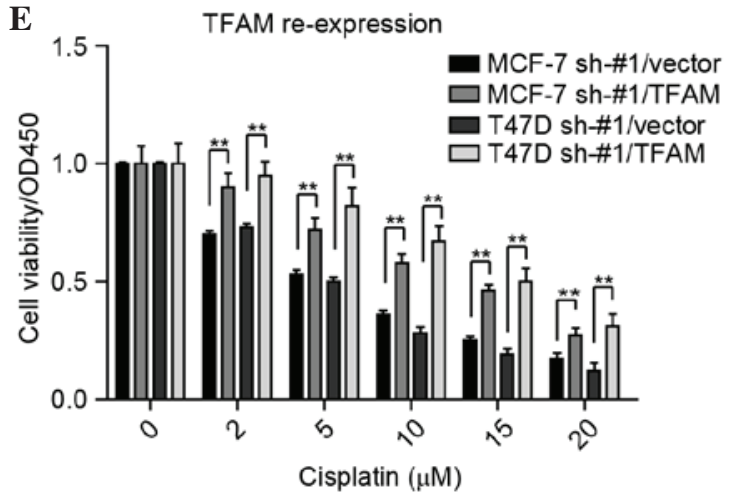

B

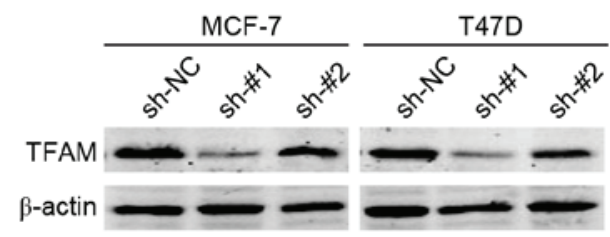

D

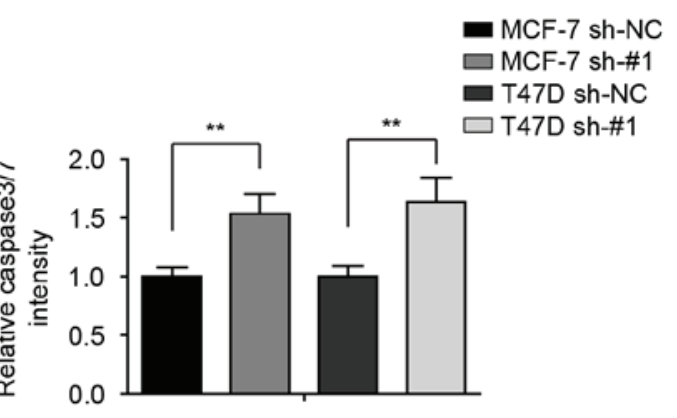

$\mathbf{F}$

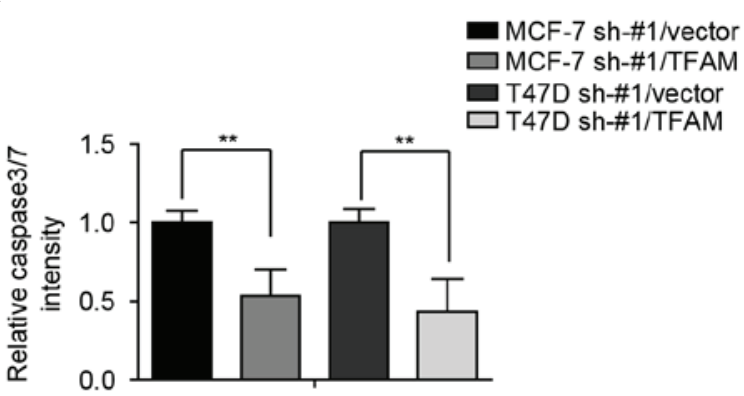

Figure 3. Silencing of TFAM in MCF-7 and T47D cells increases sensitivity to cisplatin. Alterations in the (A) mRNA and (B) protein levels in MCF-7 and MDA-MB-231 cells following silencing of TFAM by the indicated shRNAs. $\beta$-actin was used as loading control. (C) Knockdown of TFAM in ER-positiive MCF-7 and T47D breast cancer cells increased sensitivity to cisplatin. (D) Activities of caspase 3/7 were enhanced in MCF-7 and T47D cells with TFAM knockdown. (E) Reintroduction of TFAM to TFAM-knockdown cell lines rescued the increased sensitivities to cisplatin from TFAM knockdown. (F) Reintroduction of TFAM to TFAM-knockdown cell lines inhibited the activities of caspase $3 / 7$. $^{* *} \mathrm{P}<0.01$, comparison indicated by bracket. ER, estrogen receptor; TFAM, mitochondrial transcription factor A; OD, optical density; sh, short hairpin RNA; NC, negative control.

A

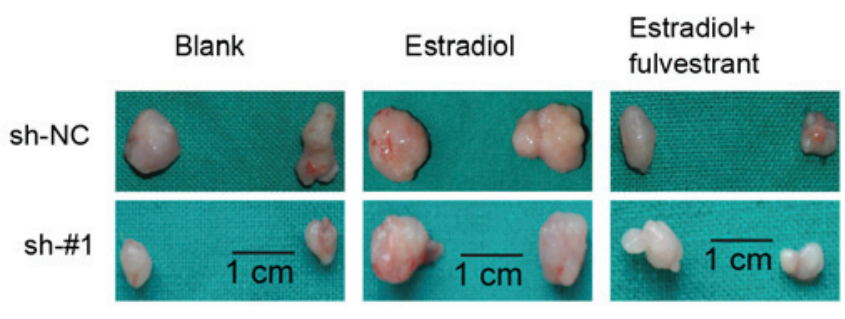

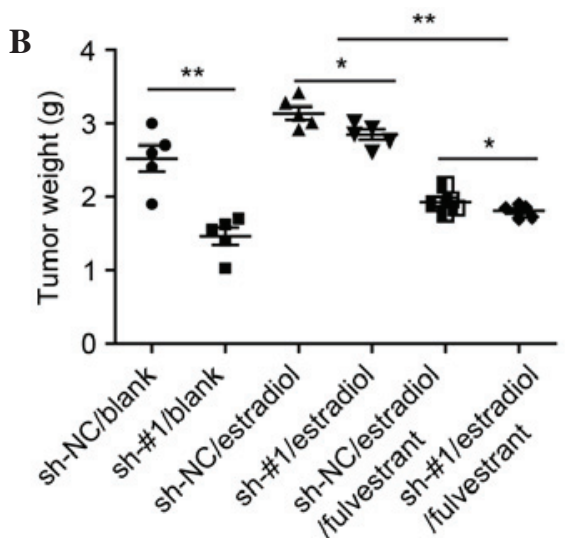

Figure 4. Silencing of mitochondrial transcription factor A increases sensitivity of MCF-7 cells to cisplatin in vivo. (A) Stably transfected MCF-7 cells were xenotransplanted into nude mice, which were then treated with estradiol or estradiol and fulvestrant every 2 days for 2 months, and then with $20 \mu \mathrm{M}$ cisplatin every 2 days for 2 months. (B) Statistical analysis of tumor weights in each group. ${ }^{*} \mathrm{P}<0.05$ and ${ }^{* *} \mathrm{P}<0.01$. sh, short hairpin RNA; NC, negative control. 
ER-positive breast cancer cell to cisplatin. For this investigation, two pairs of shRNAs (sh-\#1 and sh-\#2) specific against TFAM, were used to generate stable TFAM knockdown and control shRNA transfection in the MCF-7 and T47D cell lines. The results showed that sh-\#1 effectively knocked down TFAM in these breast cancer cell lines (Fig. 3A and B).

The present study then examined the effect of TFAM knockdown on breast cancer cell viability when treated with different concentrations of cisplatin. As shown in Fig. 3C, TFAM knockdown significantly inhibited the cell viabilities. The activities of caspase $3 / 7$ in these stable cell lines were also detected, and it was found that the cells with TFAM knockdown exhibited higher relative activities of caspase 3/7 (Fig. 3D). Furthermore, to demonstrate that the effects on the were specifically due to the silencing of TFAM, TFAM was reintroduced into the sh-\#1-transfected MCF-7 and T47D cells, and it was found that the re-expression of TFAM reversed the TFAM knockdown-induced cisplatin insult (Fig. 3E and F). Taken together, these findings indicated that TFAM may be closely associated with the sensitivity of breast cancer cells to cisplatin.

TFAM knockdown elevates sensitivity to cisplatin in ER-positive breast cancer cells in vivo. The effects of TFAM knockdown in the breast cancer cells prompted the investigation of whether TFAM had the same effect in vivo. The present study used stably-transfected MCF-7 cell lines and performed xenograft tumor growth assays. As shown in Fig. 4A and B, it was found that the knockdown of TFAM resulted in the tumor size and weight being significantly smaller and lighter, respectively, compared with the control groups when treated with estradiol. These in vivo findings confirmed that TFAM was essential in cisplatin resistance in the ER-positive breast cancer.

\section{Discussion}

In the present study, it was found that TFAM promoted ER-positive breast cancer cell survival following cisplatin treatment. Previously, it has been shown that patients with ER-positive breast cancer are more likely to exhibit the features of cisplatin resistance (23). The present study provided preliminary clues to the molecular mechanism underlying this clinical observation.

Regarding the sensitivity of breast cancer cells to cisplatin, the findings of the present study were consistent with previous evidence showing that ER-negative breast cancer cells are more sensitive to cisplatin treatment, and ER-positive breast cancer cells are more tolerant to cisplatin (24). It is known that several oncogenic genes and tumor suppressors are associated with cisplatin sensitivity, including AKT, c-myc and p53 (25-27); however, cisplatin resistance is a cell type- and tissue dependent-phenomenon, and the clinical mechanisms leading to cisplatin resistance are complex (28-30). In the present study, it was found that estrogen indirectly regulated TFAM, which may also be involved in cisplatin resistance.

ER can be activated by estrogen, and activated ER can activate the transcription of NRF1, which can regulate the expression of TFAM. TFAM is a mitochondrial transcription factor, which functions in mitochondrial transcription regulation, and also functions in mitochondrial DNA replication and repair $(10,11)$. As the molecular mechanism underlying the effect of cisplatin on cancer cell death is to disturb the double strand of DNA, including mtDNA (31), these previous studies indicate a potential mechanism by which TFAM can protect mtDNA from cisplatin-induced injury.

The in vitro and in vivo experiments performed in the present study confirmed the hypothesis that TFAM has a protective effect in ER-positive breast cancer cells exposed to cisplatin treatment. It is known that mitochondrial damage is a major trigger for the induction of cell apoptosis and necrosis (32), however, the detailed role of TFAM remains to be fully elucidated and requires further investigation.

In conclusion, the present study demonstrated that estrogen indirectly regulated the expression of TFAM to protect ER-positive breast cancer cells from cisplatin treatment. These results offer potential in guiding chemotherapy in patients with breast cancer.

\section{Acknowledgements}

This study was supported by the Shanghai Science and Technology Committee (grant no. 14ZR1408800).

\section{References}

1. DeSantis C, Ma J, Bryan L and Jemal A: Breast cancer statistics, 2013. CA Cancer J Clin 64: 52-62, 2014.

2. Kwok JM, Peck B, Monteiro LJ, Schwenen HD, Millour J, Coombes RC, Myatt SS and Lam EW: FOXM1 confers acquired cisplatin resistance in breast cancer cells. Mol Cancer Res 8: 24-34, 2010.

3. Zhang J, Wang Z, Hu X, Wang B, Wang L, Yang W, Liu Y, Liu G, Di G, Hu Z, et al: Cisplatin and gemcitabine as the first line therapy in metastatic triple negative breast cancer. Int J Cancer 136: 204-211, 2015.

4. Siddik ZH: Cisplatin: Mode of cytotoxic action and molecular basis of resistance. Oncogene 22: 7265-7279, 2003.

5. Yazlovitskaya EM and Persons DL: Inhibition of cisplatin-induced ATR activity and enhanced sensitivity to cisplatin. Anticancer Res 23: 2275-2279, 2003.

6. Wei Q, Dong G, Yang T, Megyesi J, Price PM and Dong Z: Activation and involvement of $\mathrm{p} 53$ in cisplatin-induced nephrotoxicity. Am J Physiol Renal Physiol 293: F1282-F1291, 2007.

7. Yoshida K, Ozaki T, Furuya K, Nakanishi M, Kikuchi H, Yamamoto H, Ono S, Koda T, Omura K and Nakagawara A: ATM-dependent nuclear accumulation of IKK-alpha plays an important role in the regulation of p73-mediated apoptosis in response to cisplatin. Oncogene 27: 1183-1188, 2008.

8. Pegram MD, Lipton A, Hayes DF, Weber BL, Baselga JM, Tripathy D, Baly D, Baughman SA, Twaddell T, Glaspy JA and Slamon DJ: Phase II study of receptor-enhanced chemosensitivity using recombinant humanized anti-p185HER2/neu monoclonal antibody plus cisplatin in patients with HER $2 /$ neu-overexpressing metastatic breast cancer refractory to chemotherapy treatment. J Clin Oncol 16: 2659-2671, 1998.

9. Sui M, Zhang H and Fan W: The role of estrogen and estrogen receptors in chemoresistance. Curr Med Chem 18: 4674-4683, 2011.

10. Mattingly KA, Ivanova MM, Riggs KA, Wickramasinghe NS, Barch MJ and Klinge CM: Estradiol stimulates transcription of nuclear respiratory factor-1 and increases mitochondrial biogenesis. Mol Endocrinol 22: 609-622, 2008.

11. Cam H, Balciunaite E, Blais A, Spektor A, Scarpulla RC, Young R, Kluger Y and Dynlacht BD: A common set of gene regulatory networks links metabolism and growth inhibition. Mol Cell 16: 399-411, 2004

12. Kang D, Kim SH and Hamasaki N: Mitochondrial transcription factor A (TFAM): Roles in maintenance of mtDNA and cellular functions. Mitochondrion 7: 39-44, 2007. 
13. Hallberg BM and Larsson NG: TFAM forces mtDNA to make a U-turn. Nat Struct Mol Biol 18: 1179-1181, 2011.

14. Yang Z, Schumaker LM, Egorin MJ, Zuhowski EG, Guo Z and Cullen KJ: Cisplatin preferentially binds mitochondrial DNA and voltage-dependent anion channel protein in the mitochondria membrane of head and neck squamous cell carcinoma: Possible role in apoptosis. Clin Cancer Res 12: 5817-5825, 2006.

15. Livak KJ and Schmittgen TD: Analysis of relative gene expression data using real-time quantitative PCR and the 2(-Delta Delta C(T)) Method. Methods 25: 402-408, 2001.

16. Byrski T, Huzarski T, Dent R, Gronwald J, Zuziak D, Cybulski C, Kladny J, Gorski B, Lubinski J and Narod SA: Response to neoadjuvant therapy with cisplatin in BRCA1-positive breast cancer patients. Breast Cancer Res Treat 115: 359-363, 2009.

17. Hsieh CY, Santell RC, Haslam SZ and Helferich WG: Estrogenic effects of genistein on the growth of estrogen receptor-positive human breast cancer (MCF-7) cells in vitro and in vivo. Cancer Res 58: 3833-3838, 1998.

18. Ström A, Hartman J, Foster JS, Kietz S, Wimalasena J and Gustafsson JA: Estrogen receptor beta inhibits 17beta-estradiolstimulated proliferation of the breast cancer cell line T47D. Proc Natl Acad Sci USA 101: 1566-1571, 2004.

19. Ferguson AT, Lapidus RG, Baylin SB and Davidson NE: Demethylation of the estrogen receptor gene in estrogen receptor-negative breast cancer cells can reactivate estrogen receptor gene expression. Cancer Res 55: 2279-2283, 1995.

20. Doane AS, Danso M, Lal P, Donaton M, Zhang L, Hudis C and Gerald WL: An estrogen receptor-negative breast cancer subset characterized by a hormonally regulated transcriptional program and response to androgen. Oncogene 25: 3994-4008, 2006.

21. $\mathrm{Li} \mathrm{L}$ and $\mathrm{Yu}$ AQ: The functional role of peroxiredoxin 3 in reactive oxygen species, apoptosis, and chemoresistance of cancer cells. J Cancer Res Clin Oncol 141: 2071-2077, 2015.

22. Hosoya $\mathrm{N}$ and Miyagawa K: Targeting DNA damage response in cancer therapy. Cancer Sci 105: 370-388, 2014.
23. Gonzalez-Angulo AM, Morales-Vasquez F and Hortobagyi GN: Overview of resistance to systemic therapy in patients with breast cancer. Adv Exp Med Biol 608: 1-22, 2007.

24. Silver DP, Richardson AL, Eklund AC, Wang ZC, Szallasi Z, Li Q, Juul N, Leong CO, Calogrias D, Buraimoh A, et al: Efficacy of neoadjuvant Cisplatin in triple-negative breast cancer. J Clin Oncol 28: 1145-1153, 2010.

25. Funato T, Kozawa K, Kaku M and Sasaki T: Modification of the sensitivity to cisplatin with c-myc over-expression or down-regulation in colon cancer cells. Anticancer Drugs 12: 829-834, 2001.

26. Fraser M, Bai T and Tsang BK: Akt promotes cisplatin resistance in human ovarian cancer cells through inhibition of p53 phosphorylation and nuclear function. Int J Cancer 122: 534-546, 2008 .

27. Bradford CR, Zhu S, Ogawa H, Ogawa T, Ubell M, Narayan A, Johnson G, Wolf GT, Fisher SG and Carey TE: P53 mutation correlates with cisplatin sensitivity in head and neck squamous cell carcinoma lines. Head Neck 25: 654-661, 2003.

28. Niedner H, Christen R, Lin X, Kondo A and Howell SB: Identification of genes that mediate sensitivity to cisplatin. Mol Pharmacol 60: 1153-1160, 2001.

29. Funato T, Kozawa K, Kaku M and Sasaki T: Modification of the sensitivity to cisplatin with c-myc over-expression or down-regulation in colon cancer cells. Anticancer Drugs 12: 829-834, 2001.

30. Fraser M, Bai T and Tsang BK: Akt promotes cisplatin resistance in human ovarian cancer cells through inhibition of p53 phosphorylation and nuclear function. Int J Cancer 122: 534-546, 2008.

31. Olivero OA, Semino C, Kassim A, Lopez-Larraza DM and Poirier MC: Preferential binding of cisplatin to mitochondrial DNA of Chinese hamster ovary cells. Mutat Res 346: 221-230, 1995.

32. Kroemer G, Dallaporta B and Resche-Rigon M: The mitochondrial death/life regulator in apoptosis and necrosis. Annu Rev Physiol 60: 619-642, 1998. 